\section{Myasthenia gravis is a rare but treatable disease}

To the Editor: Myasthenia gravis $(\mathrm{MG})$ is a rare but treatable disease. Early recognition and treatment can prevent mortality and morbidity, thereby reducing the burden of the disease. In $85-90 \%$ of cases, the diagnosis is made with an acetylcholine receptor antibody (AChR) radioimmunoprecipitation assay. As a diagnostic test, the assay is 99.9\% specific for the presence of MG. ${ }^{[1]}$ We recently reported the incidence of AChR-positive MG in South Africa (SA) based on all the laboratory data over a 2-year period $(2011-2012) \cdot{ }^{[2]}$ Here, we highlight two issues emanating from that research of importance to specialist services delivery and MG management.

The first issue relates to the need to improve diagnostic capacity for neurological disorders in regions with limited resources. Our results showed an annual crude incident rate of 8.5 per million for AChR-positive MG, which is comparable with the pooled estimate of 7.3 per million from a worldwide systematic study, ${ }^{[3]}$ and represents an apparent increase in incidence from 2.6 per million reported in $2004 .^{[4]}$ Rather than indicating an increased incidence, we believe the observed increase represents improved diagnostic capacity in the form of improved access to the assay, as well as better recognition of the disease. Diagnostic capacity has increased thanks to a number of SA universities establishing outreach programmes, increasing access to specialist services. Still, MG is currently diagnosed mostly in provinces with more resources (Fig. 1).

The second issue relates to the efficient use of resources in MG management. We found that $\sim 10 \%$ of requests were repeat antibody tests. Some clinicians are routinely tracking antibody titres to monitor disease activity. There is currently poor evidence to suggest that the AChR antibody test is the preferred biomarker

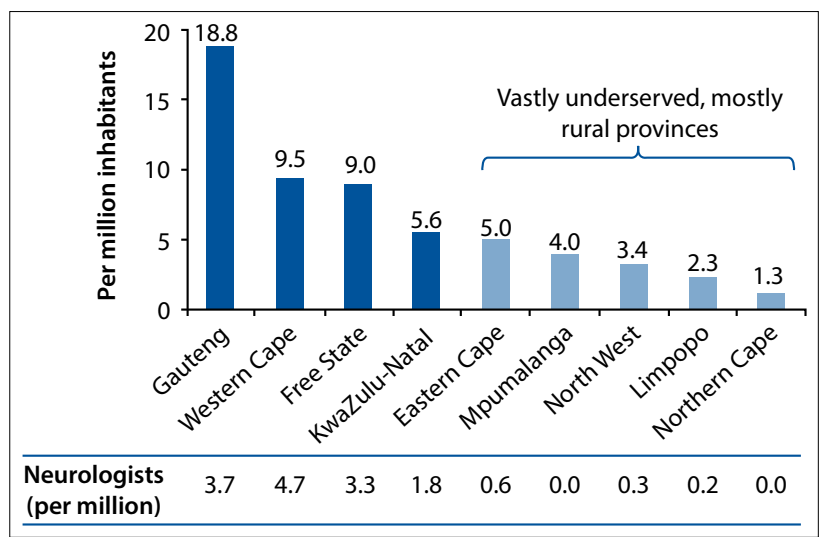

Fig. 1. Average incidence ratios for AChR-antibody-positive myasthenia gravis in SA between 2011 and 2012, by province. The number of practising neurologists per province as of December 2012 is shown. Incidence rates calculated using denominators from the 2011 census and 2012 adjusted population estimates (www.statssa.gov.sa) for disease activity compared with bedside clinical assessments. Constantly changing conditions in the laboratory, such as variations in temperature, etc., materially affect assay results. Therefore assay result $\mathrm{A}$ performed at time 1 cannot accurately be compared with assay result $B$ performed at a different time. Scientific experimental methods would require samples $A$ and $B$ to be performed in the same assay for comparative purposes. In such an experiment using stored samples collected in a prospective MG treatment trial, a fall in antibody titres correlated weakly with improvement as assessed by two validated clinical outcome measures. ${ }^{[5]}$ This study also compared serial measurements of AChR titres in an observational cohort performed at least 6 months apart and at separate times, and found even less correlation between clinical improvement and change in titres. Indeed, the conclusion was that a clinical assessment tool provided a more effective measure to monitor response to therapy. The MG composite score is a good example of a simple clinical tool that can be used to monitor patients. It is a validated instrument that takes 5 minutes to perform, and includes ocular, bulbar, respiratory and proximal muscle strength evaluations. ${ }^{[6]}$ The bulbar and respiratory items are largely based on patient symptoms. In contrast, each commercial AChR antibody test costs $\sim$ ZAR1 000.

In conclusion, our recent work suggests that the overall incidence rate of MG in our population is comparable with rates reported in North American and European studies in keeping with a biological rather than environmental aetiology for MG. The geographical variation of the incidence rate within the country underscores the importance of outreach programmes in regions with limited resources. Finally, when managing a chronic disease such as MG, it is preferable to treat the patient's symptoms rather than rely on an expensive laboratory test that cannot be accurately interpreted outside a rigorous scientific experiment.

Busi Mombaur, Jeannine M Heckmann

Neurology Research Group, Division of Neurology,

Groote Schuur Hospital and University of Cape Town, South Africa

busisiwe.mombaur@wits.ac.za

Vincent A, Clover L, Buckley C, Grimley Evans J, Rothwell PM. Evidence of underdiagnosis of myasthenia gravis in older people. J Neurol Neurosurg Psychiatry 2003;74(8):1105-1108. [http:// dx.doi.org/10.1136/jnnp.74.8.1105]

2. Mombaur B, Lesosky M, Liebenberg L, Vreede H, Heckmann J. Incidence of acetylcholine receptor positive myasthenia gravis in South Africa. Muscle Nerve 2015;51(4):533-537. [http://dx.doi. org $/ 10.1002 /$ mus.24348

3. Carr AS, Cardwell CR, McCarron PO, McConville J. A systematic review of population based epidemiological studies in myasthenia gravis. BMC Neurol 2010;10(46). [http://dx.doi. org/10.1186/1471-2377-10-46]

Bateman KJ, Schinkel M, Little F, Liebenberg L, Vincent A, Heckmann JM. Incidence of seropositive myasthenia gravis in Cape Town and South Africa. S Afr Med J 2007;97(10):959-962.

5. Sanders DB, Burns TM, Cutter GR, Massey JM, Juel VC, Hobson-Webb L; Muscle Study Group. Does canders DB, Burns TM, Cutter GR, Massey JM, Juel VC, Hobson-Webb L; Muscle Study Group. Does
change in acetylcholine receptor antibody level correlate with clinical change in myasthenia gravis? change in acetylcholine receptor antibody level correlate with clinical ch

6. Burns TM. The MG composite: An outcome measure for myasthenia gravis for use in clinical trials Burns TM. The MG composite: An outcome measure for myasthenia gravis for use in clinical trials
and everyday practice. Ann N Y Acad Sci 2012;1274:99-106. [http://dx.doi.org/10.1111/j.1749and everyday practe.

S Afr Med J 2015;105(8):619. DOI:10.7196/SAMJnew.8328 